\title{
Central diabetes insipidus (Infundibuloneuro hypophysitis): A late complication of COVID-19 infection
}

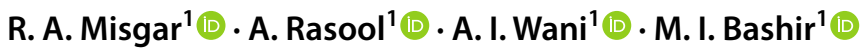

Received: 2 May 2021 / Accepted: 25 June 2021 / Published online: 3 July 2021

(C) Italian Society of Endocrinology (SIE) 2021

Keywords Diabetes insipidus · COVID-19 $\cdot$ Hypophysitis

To the Editor,

A novel coronavirus disease (COVID-19), caused by severe acute respiratory syndrome coronavirus 2 (SARS-CoV-2), has engulfed the world and has affected more than 151 million people, claiming more than 3.1 million lives across 192 countries globally. In symptomatic patients, the primary presentation is viral pneumonia sometimes complicated by acute respiratory distress syndrome. Some of the well-documented complications associated with COVID-19 infection include myocarditis, pericarditis, restrictive lung disease and thromboembolic events. However, the endocrine complications especially the hypothalamo-pituitary dysfunction of COVID-19 remains largely unexplored.

COVID-19 uses angiotensin-converting enzyme 2 (ACE2) as a receptor. In the near future, there is a strong possibility that we will encounter medium to long-term consequences of COVID-19 on the endocrine system because a number of endocrine organs express ACE2, namely pancreas, thyroid, testis, ovary, adrenal glands and pituitary [1]. However, the published data on the endocrine complications of COVID-19 is sparse, particularly isolated pituitary abnormalities. We present an interesting case of a middle-aged female who developed central diabetes insipidus (CDI) with magnetic resonance imaging (MRI) evidence of infundibuloneurohypophysitis as a long-term complication after recovering from COVID-19 infection.

A previously well 60-year-old multiparous female presented with fever $\left(99-101{ }^{\circ} \mathrm{F}\right)$, dry cough, myalgias, arthalgias, severe fatigue, anorexia and loss of taste. She was diagnosed with COVID-19 infection in view of positive reverse

R. A. Misgar

drreyaz07@rediffmail.com

1 Department of Endocrinology, Sher-i-Kashmir Institute of Medical Sciences, Srinagar, Kashmir, India transcription polymerase reaction (RT-PCR). She was hemodynamically stable with normal oxygen saturation and was treated symptomatically at home without requiring any supplemental oxygen. Her symptoms abated over a period of 10 days. Eight weeks after the diagnosis of COVID-19 infection, she presented with sudden onset polyuria, nocturia, polydipsia and craving for cold water. Her 24-h urinary volume was $6 \mathrm{~L}$. Biochemical evaluation revealed hypernatremia (serum sodium $152 \mathrm{meq} / \mathrm{dl}$ ) and normal serum potassium, creatinine, calcium and blood glucose. Paired serum and urine osmolality were $300 \mathrm{mOsm} / \mathrm{kg}$ and $177 \mathrm{mOsm} / \mathrm{kg}$, respectively, suggesting a diagnosis of diabetes insipidus. A contrast enhanced MRI revealed enlarged pituitary with an absent posterior pituitary bright spot on T1-weighted images associated with thickening of the pituitary stalk of $3.5 \mathrm{~mm}$ in transverse dimension suggestive of infundibuloneuro hypophysitis (Fig. 1). Further endocrine workup, revealed normal serum T3 $(1.52 \mathrm{ng} / \mathrm{ml})$, T4 $(9.61 \mu \mathrm{g} / \mathrm{dl})$, TSH $(3.44 \mathrm{mU} / \mathrm{L})$, morning cortisol $(21.96 \mu \mathrm{g} / \mathrm{dl})$ and prolactin $(12.7 \mathrm{ng} / \mathrm{ml})$. On the basis of clinical profile, biochemical evaluation and MRI features, a diagnosis of CDI was made. The patient was started on oral desmopressin $0.1 \mu \mathrm{g}$ twice a day to which she showed a prompt and gratifying response. She is on follow up for more than 5 months and is doing well.

In view of MRI findings, an extensive evaluation was undertaken to uncover the etiology of hypophysitis like germ cell tumor, granulomatous disease or lymphocytic hypophysitis. Complete blood counts, ESR, liver function tests, antinuclear antibody and chest radiograph were normal. Cerebrospinal fluid analysis revealed normal levels of $\alpha$-fetoprotein $(0.50 \mathrm{IU} / \mathrm{ml}$; normal range $0-16.53 \mathrm{IU} / \mathrm{ml})$ and human chorionic gonadotropin-5 $(1.72 \mathrm{mIU} / \mathrm{ml}$; normal range $0-16.53 \mathrm{mIU} / \mathrm{ml})$. Serum levels of angiotensinconverting enzyme (ACE) were normal (15.0 U/L; normal range 8-52 U/L), as was serum $\beta_{2}$ microglobulin (1926 ng/ $\mathrm{ml}$; normal range $609-2366 \mathrm{ng} / \mathrm{ml}$ ). She also had normal 


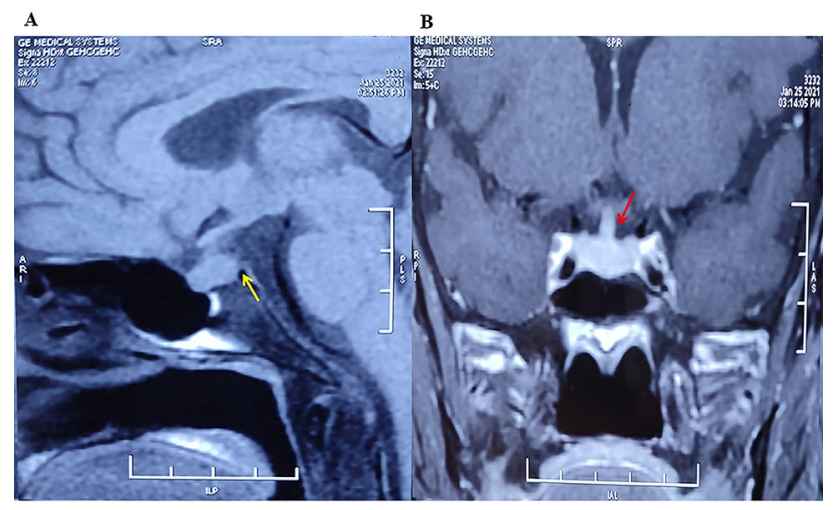

Fig. 1 A T1weighted MRI showing enlarged pituitary with absent posterior pituitary bright spot (yellow arrow); B Post contrast MRI showing thickened and enhanced pituitary stalk (red arrow)

serum levels of IgG4 with the value of $1.28 \mathrm{~g} / \mathrm{L}$ (range $0.03-2.01 \mathrm{~g} / \mathrm{L}$ ). Biopsy of hypothamic pituitary area was declined by the patient.

The clear temporal relationship of the onset of DI to COVID-19 infection and exclusion of the most common causes of hypophysitis make us believe strongly that the DI in our patient was due to COVID-19 induced hypophysitis. In addition, in our patient absence of headache/visual impairment, normoprolactinemia, eucortisolism and presence of isolated CDI make lymphocytic hypophysitis very unlikely but not impossible. The evidence of hypothalamopituitary involvement in SARS was first reported by Leow et al. in 2005 [2]. In this study involving 61survivors of SARS, $40 \%$ had evidence of central hypocortisolism and $5 \%$ had central hypothyroidism. To the best of our knowledge, there are only two published reports of COVID-19 infection-related CDI [3, 4]. Like our patient, both these cases developed CDI 4-5 weeks after COVID-19 infection. This implies that CDI is a late sequela of COVID-19 infection. In both the previously published cases, MRI of sella and suprasellar was normal. Our case is the first and so far the only, case of COVID-19 infectionrelated CDI and MRI evidence of infundibuloneuro hypophysitis.

The possibility of inflammation-mediated reversible hypophysitis or a direct hypothalamic damage are the two possible mechanisms for the hypothalamo-pituitary dysfunction [5]. SARS-CoV-2 can directly target the hypothalamic and pituitary tissues because of a high expression of ACE-2 receptors [6]. In fact, autopsy studies have reported SARS genome-mediated edema and neuronal degeneration in the hypothalamus [2].

We conclude and emphasize that isolated pituitary abnormality in the form of CDI can be a late complication of COVID-19 infection. More studies with long-term follow-up of recovered COVID-19 patients are needed to better determine post-

COVID-19 endocrine complications, clinical course, and recovery.

\section{Acknowledgements Nil.}

Funding Nil.

\section{Declarations}

Conflict of interest All authors declare that they have no conflicts of interest.

Ethical approval All procedures performed during this retrospective were in accordance with the ethical standards of the institutional and/ or research committee and with the 1964 Helsinki Declaration and its later amendments or comparable ethical standards. The ethical committee approval is not required for case reports.

Informed consent Signed consent was obtained from the patient.

\section{References}

1. Liu F, Long X, Zhang B, Zhang W, Chen X, Zhang Z (2020) ACE2 expression in pancreas may cause pancreatic damage after SARS-CoV-2 infection. Clin Gastroenterol Hepatol 18:2128-2130

2. Leow MKS, Kwek DSK, Ng AWK, Ong KC, Kaw GJL, Lee LSU (2005) Hypocortisolism in survivors of severe acute respiratory syndrome (SARS). Clin Endocrinol (Oxf) 63:197-202

3. Rajevac H, Bachan M, Khan Z (2020) Diabetes insipidus as a symptom of covid-19 infection: case report. Chest 158:A2576

4. Sheikh AB, Javed N, Sheikh AA, Upadhyay S, Shekhar R (2021) Diabetes insipidus and concomitant myocarditis: a late sequelae of COVID-19 infection. J Investig Med High Impact case Rep 9:1-4

5. Pal R, Banerjee M (2020) COVID-19 and the endocrine system: exploring the unexplored. J Endocrinol Invest 43:1027-1031

6. Marazuela M, Giustina A, Puig-Domingo M (2020) Endocrine and metabolic aspects of the COVID-19 pandemic. Rev Endocr Metab Disord 21:495-507

Publisher's Note Springer Nature remains neutral with regard to jurisdictional claims in published maps and institutional affiliations. 